\title{
Temporal Lobe Epilepsy Associated with Cerebral Venous Angioma
}

\author{
-Case Report- \\ Masami FujII, Tetsuhiro KITAHARA, Junta MOROI, Shoichi KATO, \\ and Haruhide ITO
}

Department of Neurosurgery, Yamaguchi University School of Medicine, Ube, Yamaguchi

\begin{abstract}
A 21-year-old male presented with temporal lobe epilepsy associated with a venous angioma in the ipsilateral frontal lobe, presenting as intractable complex partial seizures. Neuroimaging showed a cerebral venous angioma in the right dorsolateral and opercular frontal lobe, and atrophy of the right hippocampus. As the ictal electroencephalogram (EEG) obtained with subdural electrodes indicated spike discharges initiating from the right mesial temporal lobe, temporal lobectomy was performed. The patient was seizure-free after the operation. Patients with epilepsy who have a cerebral venous angioma require precise analysis of the seizure pattern and an ictal EEG because a cerebral venous angioma may be associated with an another epileptogenic lesion which is surgically treatable.
\end{abstract}

Key words: cerebral venous angioma, temporal lobe epilepsy, ictal electroencephalogram, temporal lobectomy

\section{Introduction}

Cerebral venous angiomas consist of multiple, radially oriented, dilated medullary veins, and drain into the transparenchymal venous system. These lesions were thought to be rare. ${ }^{15)}$ However, venous angiomas may be the most common form of vascular malformation, accounting for $63 \%$ of 165 malformations found in 4069 consecutive autopsies. ${ }^{11]}$ Cerebral venous angiomas are usually found incidentally, and are rarely associated with hemorrhage or seizures, ${ }^{5,7,9,13)}$ In contrast, the incidence of seizures associated with symptomatic cerebral venous angiomas ranges from $8 \%$ to $29 \% .^{3.5-8,12)}$ Epileptogenic foci are most commonly located in the temporal lobe, and manifest clinically as temporal lobe epilepsy. Hippocampal sclerosis is the most common cause of intractable temporal lobe epilepsy. Although venous angiomas and temporal lobe epilepsy caused by hippocampal sclerosis are individually common, temporal lobe epilepsy associated with venous angioma seems to be unusual. Neuroimaging cannot determine whether the epileptic focus is an area of hippocampal sclerosis or a venous angioma.

Received April 15, 1997; Accepted March 27, 1998
We describe a case of temporal lobe epilepsy associated with venous angioma successfuly treated by surgery.

\section{Case Report}

A 21-year-old male was admitted for surgical treatment of intractable seizures, which had started at age 13 years. Administration of adequate anticonvulsants (phenytoin $300 \mathrm{mg}$ and carbamazepine $800 \mathrm{mg}$ per day) had failed to prevent complex partial seizures two to three times per month, accompanied by generalized tonic-clonic convulsions once every 3 months. His psychomotor development was normal, and his personal and family history was unremarkable.

Neurological examinations revealed no notable abnormalities. $\mathrm{T}_{1}$-weighted magnetic resonance (MR) imaging showed multiple flow voids in the right frontal lobe and atrophy of the right hippocampus and right cerebral hemisphere compared to the left side (Fig. 1). Cerebral angiography showed abnormal veins resembling caput medusae in the right frontal lobe and a dilated right sylvian vein (Fig. 2). The diagnosis was cerebral venous angioma based on these angiographic findings. The intracarotid amytal test 

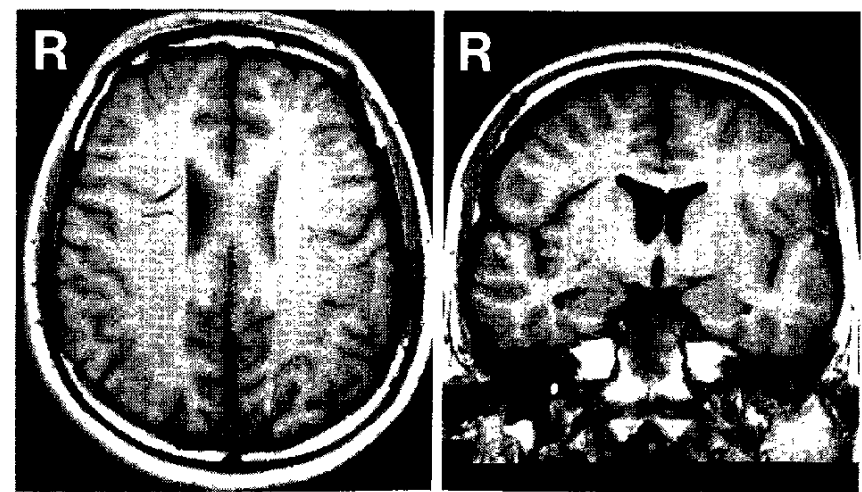

Fig. $1 \mathrm{~T}_{1}$-weighted magnetic resonance images, axial view (left) showing linear flow voids in the right frontal lobe, and coronal view (right) showing atrophy of the right hippocampus, and dilatation of the right anterior and inferior horns of the lateral ventricle.

revealed that the left hemisphere was the dominant side for speech. Neuropsychological examination showed the patient's intelligence quotient (IQ) as assessed by the Wechsler Adult Intelligence ScaleRevised was 85 (verbal IQ 81, performance IQ 96).

His seizure history and data obtained by long-term video/electroencephalography (EEG) recording suggested the main type of seizure was complex partial seizure. The seizures involved a déjà vu sensation, motionless stare, involuntary oroalimentary movement, and loss of consciousness, followed by gener-

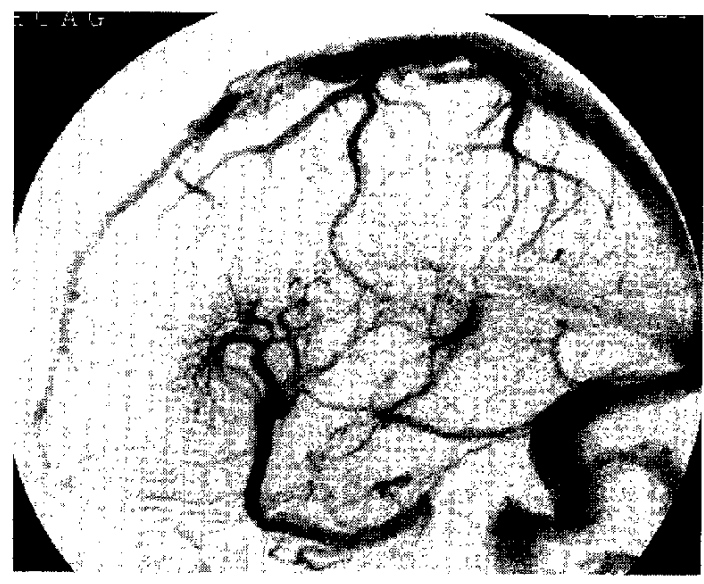

Fig. 2 Cerebral angiogram, venous phase, showing an abnormal vein resembling a caput medusae and a dilated sylvian vein in the right frontal lobe.

alized tonic-clonic convulsion. Interictal scalp EEG revealed sporadic spikes in the right frontotemporal region. However, as the decision of seizure focus was difficult from only scalp EEG, long-term invasive video/EEG recording with subdural electrodes was performed. The electrodes were placed on the right frontal lobe, where the cerebral venous angioma was located, on the right mesiobasal temporal lobe, and on the lateral surface of the right temporal lobe. Spike discharges started from the right mesial temporal lobe, then spread to the whole of the right temporal lobe (Fig. 3), and finally to the

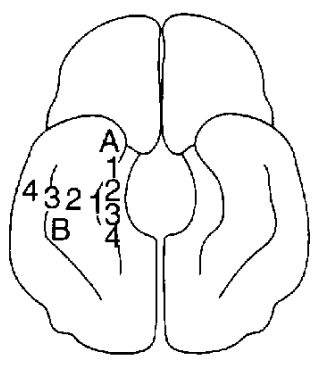

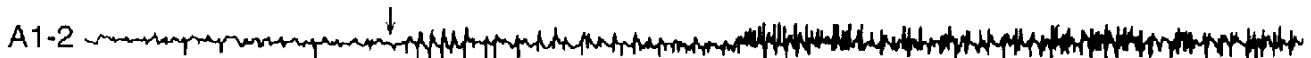

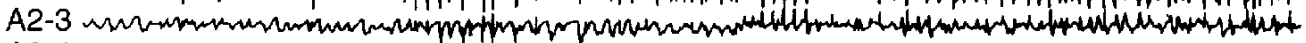
A3-4

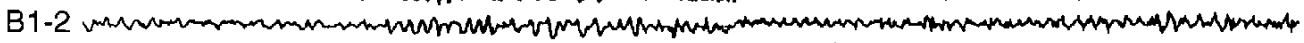

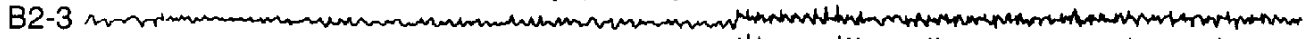
B3-4 wer row C1-2 C2-3

C3-4

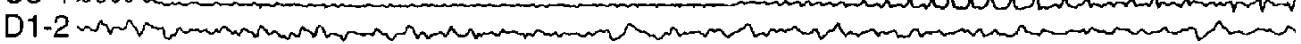
D2-3

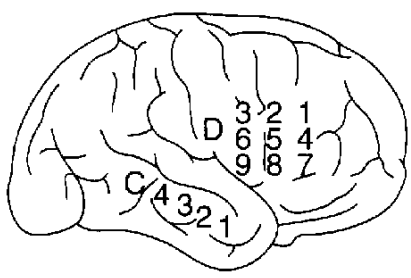

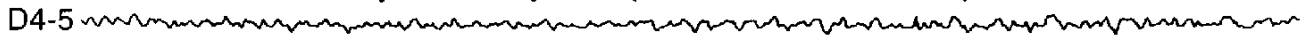

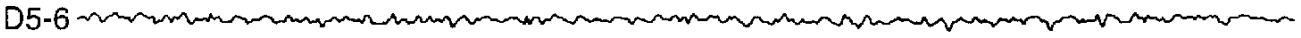
D7-8 D8-9

Fig. 3 Ictal electroencephalograms recorded with subdural electrodes showing spike discharges start from the right mesial temporal lobe and spread to the right temporal lobe, but no spike discharge from the right frontal lobe. The arrow indicates the onset of symptoms. 
right frontal lobe. When the spike discharges spread to the right frontal lobe, tonic-clonic convulsion was observed. Based on the patient's symptoms and the results of subdural EEG recording, the seizure focus was considered to be the right mesial temporal region.

Anteromesiotemporal lobectomy was performed. After resection of $4 \mathrm{~cm}$ of the anterior temporal neocortex, the hippocampus and medial structures were resected. Histological examination of the resected hippocampus revealed neuronal cell loss and mild gliosis, predominantly in the CA1 region. No remarkable histological changes were found in the resected temporal cortex.

The patient has been seizure-free for 14 months after surgery and requires smaller dosage of anticonvulsants than before surgery. Scalp EEG obtained after surgery showed disappearance of the spike discharges.

\section{Discussion}

The most common angiographic features of venous angiomas are deep medullary veins with a caput medusae appearance, accompanied by a collecting vein draining into the superficial cortical veins or the dural sinus. Computed tomography shows venous angioma as a region of linear enhancement in the deep white matter, and MR imaging shows a flow void. ${ }^{1,3,7,8,12,14)}$ The criteria for MR imaging of mesial temporal sclerosis are anterior temporal lobe atrophy, asymmetry of the temporal horns, hippocampal atrophy, and increased signal intensity from the hippocampus on $\mathrm{T}_{2}$-weighted images. ${ }^{6)}$ As our patient had both cerebral venous angioma and mesial temporal sclerosis, neuroimaging evidence of the epileptic focus was difficult to pinpoint from only the neuroimages.

EEG and video recording are the most essential monitoring tools for the diagnosis and identification of epileptic foci. Recent developments have allowed viewing of the seizure and EEG easily on the same display device and storage of the data on videotape. The features of temporal lobe epilepsy are well known and involve simple partial or complex partial seizures followed by secondary generalized convulsions. ${ }^{21}$ Simple partial seizures are typically characterized by autonomic signs or symptoms and psychic symptoms. Complex partial seizures begin with motor arrest followed by oroalimentary automatism. Cerebral venous angiomas are usually associated with generalized seizures, ${ }^{1,4,8,15)}$ but some patients experience jacksonian march, ${ }^{131}$ partial seizures ${ }^{87}$ and complex partial seizures. ${ }^{1)}$ However, the precise features of these seizures have not been de- scribed.

In our patient, the cerebral venous angioma was located in the opercular and dorsolateral areas of the right frontal lobe. A revised classification of epilepsies and epileptic syndromes ${ }^{21}$ shows the characteristics of seizures arising from the dorsolateral frontal lobe are tonic or, less commonly clonic seizures with versive eye and head movements, and speech arrest. The characteristics of seizures arising from the opercular area are mastication, salivation, swallowing and laryngeal symptoms, speech arrest, epigastric aura, fear, and autonomic phenomena. Clonic facial movements, secondary sensory symptoms including numbness (particularly in the hands), and abnormal movement of both arms may also be seen. The seizures in our patient originated from the mesial temporal lobe, as he had the typical pattern of mesial temporal lobe seizures, including psychic symptoms (déjà vu) and motor arrest followed by oral automatism. EEG gave useful information for identifying the seizure focus, but interictal EEG is not always reliable. Our patient had localized spikes in the right frontotemporal area, but correct identification of the seizure focus was difficult based on only scalp EEG recording, and the epileptic focus was finally identified from an ictal EEG obtained with subdural electrodes. There is no correlation between the location of a cerebral venous angioma and the appearance of seizures. ${ }^{1)}$ Therefore, an ictal EEG, especially recorded with intracranial electrodes, is essential for detecting the epileptic focus.

Temporal lobectomy is a common choice for treatment of patients with intractable temporal lobe epilepsy. However, many patients with cerebral venous angiomas associated with seizures have been treated using anticonvulsants. ${ }^{5,8,9,15)}$ The natural history of cerebral venous angioma is relatively benign, and surgical resection of the lesion is rarely indicated. ${ }^{53}$ Our strategy for treating the present patient was temporal lobectomy for the temporal lobe epilepsy, but no surgical treatment was planned for the frontal venous angioma. Once the focus had been confirmed to lie in the temporal lobe, temporal lobectomy was performed. Patients with venous angioma associated with epilepsy require precise analysis of the seizure pattern and EEG findings, because another epileptogenic lesion may be present which is surgically treatable.

The relationship between the venous angioma and hippocampal atrophy is unclear in this case. The etiology of venous angiomas may involve an intrauterine accident occurring during the formation of the medullary veins or their tributaries, and involving either thrombosis or some other unknown mechan- 
ism, resulting in a collateral pathway to drain the affected area of the brain. ${ }^{10}$ In our patient, the right cerebral hemisphere might have been influenced by ischemic damage at the embryonic stage, thus leading to the formation of a venous angioma and atrophy of the right cerebral hemisphere after birth. The right hippocampus, also damaged by ischemia, would have developed abnormally after birth, resulting in atrophy and the development of epileptogenicity.

\section{References}

1) Agnoli AL, Hildebrandt G: Cerebral venous angiomas. Acta Neurochir (Wien) 78: 4-12, 1985

2) Commission on Classification and Terminology of the International League Against Epilepsy: Proposal for revised classification of epilepsies and epileptic syndromes. Epilepsia 30: 389-399, 1989

3) de Slegte RGM, Kaiser M, Valk J, Polman C: Cerebral venous angiomas: $\mathrm{A}$ review of clinical and radiological features of the literature and six additional cases. J Med Imaging 1: 15-25, 1987

4) Ferrante L, Palma L, d'Addetta R, Mastronardi L, Acqui $M$, Fortuna A: Intracranial cavernous angioma. Neurosurg Rev 15: 125-133, 1992

5) Garner TB, Curling OD Jr, Kelly DL Jr, Laster W: The natural history of intracranial venous angiomas. $J$ Neurosurg 75: 715-722, 1991

6) Kuzniecky R, Suggs S, Gaudier J, Faught E: Lateralization of epileptic foci by MRI in temporal lobe epilepsy. J Neuroimaging 1: 163-167, 1991

7) Moritake $K$, Handa $H$, Mori $K$, Ishikawa $M$, Morimoto $M$, Takebe $Y$ : Venous angiomas of the brain. Surg Neurol 14: 95-105, 1980

8) Numaguchi $Y$, Kitamura $K$, Fukui $M$, Ikeda J, Hasuo K, Kishikawa T, Okudera T, Uemura K, Matsuura K: Intracranial venous angiomas. Surg Neurol 18: 193202, 1982

9) Rigamonti D, Spetzler RF, Medina M, Rigamonti K, Geckle DS, Pappas C: Cerebral venous malformations. J Neurosurg 73: 560-564, 1990

10) Saito $Y$, Kobayashi N: Cerebral venous angiomas. Clinical evaluation and possible etiology. Radiology 139: 87-94, 1981

11] Sarwar M, McCormick WF: Intracerebral venous angiomas: Case report and review. Arch Neurol 35: 323325,1978

12) Toro VE, Geyer CA, Sherman JL, Parisi JE, Brantley MJ: Cerebral venous angiomas: MR findings. J Comput Assist Tomogr 12: 935-940, 1988

13) Truwit CL: Venous angioma of the brain: History, significance, and imaging findings. AJR Am J Roentgenol 159: 1299-1307, 1992

14) Valavanis A, Wellauer J, Yasargil MG: The radiological diagnosis of cerebral venous angioma: Cerebral angiography and computed tomography. Neuroradiology 24: 193-199, 1983

15) Wendling LR, Moore JS Jr, Kieffer SA, Goldberg HI, Latchaw RE: Intracerebral venous angioma. Radiology 119: 141-147, 1976

Address reprint requests to: M. Fujii, M.D., Department of Neurosurgery, Yamaguchi University School of Medicine, 1144 Kogushi, Ube, Yamaguchi 755-8505, Japan. 